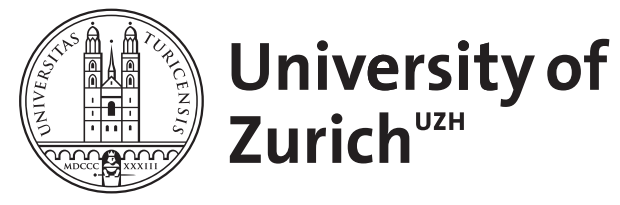

\title{
S3-Leitlinien Periimplantäre Erkrankungen: Entscheidungshilfe für den Praktiker
}

\author{
Solderer, Alex ; Schmidlin, Patrick
}

DOI: https://doi.org/10.1007/s13279-019-0077-1

Posted at the Zurich Open Repository and Archive, University of Zurich ZORA URL: https://doi.org/10.5167/uzh-194439

Journal Article

Accepted Version

Originally published at:

Solderer, Alex; Schmidlin, Patrick (2020). S3-Leitlinien Periimplantäre Erkrankungen: Entscheidungshilfe für den Praktiker. Junge Zahnarzt, Der:42-45.

DOI: https://doi.org/10.1007/s13279-019-0077-1 


\section{Auf den Punkt gebracht}

\section{Kommentar zu den S3-Leitlinien: Periimplantäre Erkrankungen}

Periimplantäre Mukositis und Periimplantitis stellen in der heutigen Praxis eine nicht seltene Herausforderung dar. In der Literatur spricht man von 43\% Mukositis und 22\% Periimplantitis gemessen auf alle eingeschlossenen Implantate (Derks et al. 2015). Betrachtet man die Anzahl der Implantationen in Deutschland, macht es auch für den Allgemeinzahnarzt Sinn, sich damit intensiv auseinanderzusetzen.

Dr. Alex Solderer, Prof. Dr. Patrick R. Schmidlin // Universität Zürich

Die Deutsche Gesellschaft zur Zahn-, Mund- und Kieferheilkunde (DGZMK) hat es sich mit ihren S3-Leitlinien zu periimplantären Erkrankungen, welche im Jahr 2016 erschienen sind, zum Ziel gesetzt, dem Kliniker eine Entscheidungshilfe zu geben. Daneben wurde im Jahr 2017 eine neue Klassifikation parodontaler und periimplantärer Erkrankungen am Weltworkshop erarbeitet und 2018 veröffentlicht. Erstmalig wurden auch die periimplantäre Gesundheit sowie die Erkrankungsformen beschrieben. Ziel dieser Betrachtung soll deshalb aus aktuellem Anlass auch sein, mögliche Überschneidungen aber vielleicht auch Widersprüche für den Praktiker zusammenzufassen und zu diskutieren.

Die pathologischen Veränderungen, auf welche die Leitlinien eingehen, betreffen die Gewebe um Implantate. Dabei entspricht die Mukositis einer Entzündung, die sich auf das suprakrestale Weichgewebsinterface beschränkt, während eine Periimplantitis auch das knöcherne Implantatlager miteinschließt und zu Knochenabbau führt. Dabei sind gewisse Ähnlichkeiten (aber auch Unterschiede) der analogen Erkrankungsformen um Zähne, sprich Gingivitis und Parodontitis, nicht ganz abzusprechen (1). Im Gegensatz zu Gingivitis und Parodontitis fehlen allerdings klare und einheitliche diagnostische Kriterien, ab wann man eindeutig von einer Periimplantitis sprechen kann, sowie klare Therapieempfehlungen in der Literatur (2). 


\section{Exkurs: Grundlagen Anatomie \& Pathophysiologie}

Um peri-implantäre Erkrankungen zu verstehen, ist es nötig, besondere anatomische und physiologische Unterschiede des Implantats im Vergleich zum Zahn zu kennen, u.a. um den protrahierten Verlauf und die gesteigerte Komplexität der Behandlung einer Periimplantitis gegenüber einer Parodontitis zu verstehen.

Mit Blick auf die Verankerung im Knochen unterscheidet sich das Implantat vom Zahn vor allem dadurch, dass bei Letzterem das durchblutete und innervierte Desmodont diese Aufgabe gelenkartig übernimmt, während wir beim Implantat typischerweise von einer Osseointegration sprechen und dabei den direkten funktionellen Kontakt zwischen Implantat-Oberfläche und Knochen meinen.

Der supra-alveoläre Weichgewebskomplex ist bei Implantaten ebenfalls nicht identisch mit demjenigen von Zähnen:

- $\quad$ Das Saumepithel ist länger

- $\quad$ In aller Regel gibt es keine in die Implantat-Oberfläche inserierenden SharpeyFasern

- $\quad$ Der Faserverlauf im Bindegewebe und die Dichte sind anders (entspricht eher einem Narbengewebe)

- $\quad$ Die biologische Breite ist insgesamt etwas ausgeprägter

Alldies hat Einfluss auf die Entzündungsantwort. Auf der einen Seite scheinen die Gewebe bei initialen Entzündungen (zum Beispiel experimentelle Mucositis) weniger stark auf den bakteriellen Reiz zu reagieren, was dazu führt, dass das Infiltrat weniger Volumen besitzt als dasjenige parodontal erkrankter Zähne, da wahrscheinlich die Durchblutung im Sinne eines eher derben narbigen Gewebes nicht vergleichbar zu reagieren vermag. Hingegen ist die Ausprägung der Entzündung bei Persistenz und Progression zu einer Periimplantitis wiederum viel stärker zu sein, was sich in einem vergleichbar grösseren Infiltratdurchmesser äussert. Zudem fehlt eine intakte gesunde trennende Gewebeschicht bei Implantaten; das entzündliche Infiltrat tritt in direkten Kontakt zum Knochen.

Zuletzt besteht auch ein starker Unterschied in der makroskopischen, wie mikroskopischen Morphologie. Während wir am Zahn eine einigermassen glatte Oberfläche vorfinden, besteht das Implantat je nach Fabrikat aus mehr oder weniger ausgeprägten Windungen mit einer mikrorauen Oberfläche. Diese Morphologie bietet zwar Vorteile im Rahmen der Einheilung, aber andererseits auch Nachteile, sobald das Implantat von Bakterien besiedelt wurde und gereinigt werden muss (3). 


\section{Ätiologie und Risikofaktoren}

Der Biofilm bleibt weiterhin der auslösende Hauptfaktor für periimplantäre Erkrankungen (4), wobei sich eine Mukositis bereits nach kurzer Zeit ungestörter Plaqueakkumulation manifestieren kann (5). Dabei kommt es im Falle einer unbehandelten Mukositis in ca. 44\% der Fälle zu einer Periimplantitis. Falls die Mukositis behandelt wird, wurde in $18 \%$ der Fälle eine Progression zu einer Periimplantitis festgestellt (5).

Vor allem eine Parodontitis-Historie oder sogar eine noch aktive parodontale Erkrankung, sowie eine schlechte Mundhygiene gelten daher als größte Risikofaktoren für die Entstehung einer periimplantären Erkrankung. Gemäß S3-Leitlinie spielt vor allem auch das Rauchen (6) eine Rolle. Der Einfluss von Diabetes und Geschlecht weisen hingegen eine ungenügende Evidenzlage auf (7). Betrachtet man die neue Klassifikation, so bestehen sowohl für das Rauchen als auch den Diabetes eine unklare Studienlage, wobei der Einfluss des Geschlechts gar nicht erwähnt wird.

Auch iatrogene Faktoren, wie eine insuffiziente oder nicht-reinigbare Versorgung oder eine Fehlpositionierung begünstigen die Entstehung einer Entzündung des periimplantären Gewebes (8). So nimmt die S3-Leitlinie lokale Faktoren, wie die Implantatposition (vor allem im Oberkiefer; (9); (2), das Fehlen einer keratinisierten Mukosa (10), festsitzenden Zahnersatz (2), vorhandene Zementreste und knöcherne Defekte sowie Dehiszenzen am Implantat (2) in die Liste möglicher Risikofaktoren auf. Als wesentlicher Unterschied zur aktuellen Klassifikation, welche sich vor allem auf eine systematische Übersichtarbeit von Wennström \& Derks (11) beruft, kann hingegen auch bei Abwesenheit von keratiniserter Mukosa ein gesundes periimplantäres Gewebe erhalten werden. Vor allem zur Steigerung des Patientenkomforts im Zuge der häuslichen Mundhygiene kann die Etablierung einer keratiniserten Mukosa aber trotzdem nötig werden.

\section{Befunderhebung}

Ein asymptomatisches Implantat lässt nicht automatisch auf Gesundheit schließen (2), wobei Schmerzen hingegen auf eine progrediente Periimplantitis hindeuten können (12).

Die klinische Befundaufnahme ähnelt der parodontalen Untersuchung an Zähnen. Hierbei liefern die Parodontalsonde und Röntgenbilder immer noch die wichtigsten Informationen. 
Vor allem die Blutung auf Sondierung (BOP) gilt als Schlüsselparameter, um ein gesundes von einem erkrankten Implantat zu unterscheiden. Somit ist eine Sondierung erforderlich, wobei allerdings auch hierbei das Risiko besteht, falsche Werte zu messen, da anatomische Besonderheiten wie zum Beispiel Windungen, Stufen am Abutment-Implantat-Interface, aber auch Zementreste oder Konkremente, das Sondieren erschweren können. Um fehlerhafte Sondierungswerte zu vermeiden, wird daher empfohlen, die Suprakonstruktion, sofern möglich, abzunehmen (13), was auch die Behandlung - wenn nötig - erleichtert (s. unten).

Vor allem die Zunahme der periimplantären Sondierungstiefe (ST) ist ein sehr zuverlässiges Zeichen, wobei diese Bewertung im Vergleich mit den Ausgangswerten geschehen muss (4). Auch dabei sind Messunterschiede zwischen gleichen und unterschiedlichen Behandlern möglich und zu berücksichtigen. Eine regelmäßige Schulung des Praxispersonals wäre diesbezüglich sicherlich sinnvoll. Im Unterschied zum Zahn kann man ohne deutliche Referenz zum Ausgangswert auch nicht unbedingt auf eine ST-Zunahme, einen Knochenabbau oder eine Schwellung schließen. Weder die S3-Leitlinien noch die aktuelle Klassifikation geben einen einheitlichen Grenzwert bei Sondierungstiefen an. Deshalb wird empfohlen, eine radiologische Referenzaufnahme bei Eingliederung der Suprakonstruktion anzufertigen.

Radiologischer Knochenabbau grenzt die Mukositis eindeutig von der Periimplantitis ab, allerdings muss von einem physiologischen Knochenumbau (Remodelling) unterschieden werden (2). Letzteres kann von der Art des Implantates, der Qualität des Implantat-Abutment-Interfaces und der Schleimhautdicke abhängen.

Eine Periimplantitis führt in 55\% zu zirkumferentiellen Klassse-I-Knochendefekten (14). Daher sehen die S3-Leitlinien eine 3D-Bildgebung als zusätzliches verlässliches diagnostisches Verfahren, das dem Zahnarzt wichtige Informationen über die Defektkonfiguration liefert (15). Hierzu sollte hinsichtlich Strahlenbelastung aber auch immer das ALARA-Prinzip („As-low-as-reasonably-achievable“) angewandt werden.

Eine mikrobiologische Diagnostik wird bei Implantaten nicht a priori empfohlen, da das Keimspektrum hier v.a. auf paropathogene Keime ausgelegt ist. Periimplantitis weist hingegen zumeist eine Mischinfektion auf (16). Ob allerdings bei Parodontitisassoziierten Fällen eine entsprechende Zusatzbefunderhebung nicht auch Vorteile haben kann, bleibt wohl kontrovers und muss noch in weiteren Studien untersucht werden (17). 
Derzeit verfügbare immunologische Analysen, die momentan vorwiegend auf Bestimmung von Zytokinen wie TNFalpha \& IL1beta beruhen, können in periimplantärer Sulkusflüssigkeit durchgeführt werden. Diese lassen aber keine Unterscheidung zwischen Mukositis und Periimplantitis zu (18). Auch der Konsenusbericht zur aktuellen Klassifikation sieht derzeit noch wenig Evidenz zu individuellen Analysen hinsichtlich Mikrobiologie oder proinflammatorischer Zytokine vorhanden, die entsprechende Tests in der Praxis rechtfertigen.

\section{Mukositis-Therapie}

Die Therapie einer periimplantären Mukositis hat zum Ziel, die klinische Entzündung im Rahmen der oben beschriebenen Infektion zu beseitigen. Hierzu muss vor allem regelmäßig eine mechanische Plaque-Entfernung erfolgen (19). Die Optimierung der häuslichen Mundhygiene kann den Therapieerfolg positiv beeinflussen(20). Eine vollständige Abheilung der peri-implantären Mukositis kann jedoch nicht bei allen Patienten vorhersehbar erreicht werden (19). Daher sollten regelmäßige Nachkontrollen (z.B. alle 3 Monate) zur frühzeitigen Erkennung des Bedarfs einer Nachbehandlung eingeplant werden. Was die Art der Instrumentierung betrifft, so gibt es vor allem für alternative Monotherapien mit Er:YAG-Laser und Air-Polishings eine gewisse Evidenz. Grundsätzlich sind bei Implantaten Alternativen wünschenswert, die zwar effektiv Biofilme und - wenn vorhanden - Konkremente oder Zementreste entfernen, jedoch die koronalen maschinierten und meist glatten Implantatoberfläche nicht beschädigen und zum Beispiel zerkratzen. Da mögliche makro- und mikroskopische Nischen die effektive Reinigung gerade bei Implantaten oft erschweren, werden nach adjuvanten Therapieformen wie zum Beispiel der Einsatz lokaler Antibiotika, Chlorhexidin-Chips und/oder die photodynamische Therapie gesucht, welche auch einen gewissen Zusatznutzen zeigen. Die Gabe systemischer Antibiotika zeigte bis dato keinen wissenschaftlichen Mehrnutzen und sollte vor allem bei lokalen Defekten nicht gedankenlos eingesetzt werden.

\section{Periimplantitis-Therapie}

Die Läsion sollte frühzeitig einer chirurgischen Therapie zugeführt werden, da bis dato mit einer nicht-chirurgischen Therapie in den meisten Fällen keine ausreichende Remission erreicht werden kann. Allerdings wird vorgängig trotzdem eine konservative 
Therapie mit kurzer Heilungsphase empfohlen, um die akute Entzündung zu reduzieren und die Gewebe zu konditionieren.

Die Art des chirurgischen Protokolls kann zum gegenwärtigen Zeitpunkt aus der Literatur leider immer noch nicht abgeleitet werden (21). Grundsätzlich steht die Dekontamination der exponierten Implantatoberflächen durch chirurgisch erleichterten Zugang im Mittelpunkt.

Auch hier lässt die Studienlage derzeit keine eindeutige Empfehlung bezüglich einer spezifischen Reinigungsmethode zu. Es stehen jedoch diverse mechanische und physikalische Methoden zur Biofilmreduktion zur Verfügung. Auch chemische Methoden zur zusätzlichen Reduktion des Biofilms können kombiniert angewendet werden (21). Ob „Vieles“ viel hilft, ist aber im Rahmen möglicher Schäden an Implantat und Gewebe für den jeweiligen Fall individuell abzuschätzen.

Eine Explantation muss bei Implantatlockerung und kann bei nicht beherrschbaren technischen Komplikationen, komplexen nicht reinigbaren Implantatdesigns (z.B. Hohlzylinder), Therapieresistenz, möglichem Übergreifen der Infektion auf anatomische Nachbarstrukturen inkl. Zähne oder bei allgemeinmedizinischer Indikation erfolgen (22).

\section{Fazit}

Periimplantäre Erkrankungen sind keine Seltenheit in der täglichen Praxis. Zweifelsohne sind eine strikte Zahnprophylaxe und entsprechende zahnerhaltende Maßnahmen, welche überhaupt das Setzen von Implantaten auf ein Minimum beschränken oder hinauszögern, die beste Periimplantitis-Prophylaxe. Sind Implantate indiziert und gesetzt, ist auch hier vor allem der Prophylaxe sowie Früherkennung und Behandlung einer Mukositis in den Vordergrund zu stellen. Die akkurate Diagnostik mit Parodontal-Sonde kombiniert mit einem radiologischen Verlaufsbefund gehört zu jeder Diagnosestellung.

Empfehlungen hinsichtlich einer chirurgischen Periimplantitis-Behandlung kann auch die Leitlinie keine bieten. In jedem Falle steht die strikte Biofilm-Reduktion/-Elimination sowie -Kontrolle ähnlich wie bei der Parodontitis-Behandlung an oberster Stelle. Allerdings erschweren diverse Implantatformen, Oberflächen und Abtumentkonfigurationen die effiziente Reinigung und vergleichbare Studienresultate. Hier ist noch viel Arbeit zu leisten. Die Leitlinie hat noch bis zum Jahr 2021 ihre Gültigkeit, aber eine Überarbeitung durch die DGI darf bereits im nächsten Jahr 
erwartet werden. Tabelle 1 fasst die derzeit gesicherten Aussagen gemäß der Leitlinie nochmals zusammen.

\begin{tabular}{|c|c|c|}
\hline & Autor & Aussage \\
\hline \multirow[t]{3}{*}{$\begin{array}{l}\text { Ätiologie \& } \\
\text { Risikofaktoren }\end{array}$} & $\begin{array}{ll}\text { Lang et al. } \\
2011\end{array}$ & $\begin{array}{ll}\text { - } & \text { Biofilm ist der auslösende Faktor für } \\
& \text { Periimplantitis }(\mathrm{PI})\end{array}$ \\
\hline & $\begin{array}{lll}\text { Salvi et al. } \\
2012\end{array}$ & $\begin{array}{l}\text { - Biofilm-Akkumulation führt zu Mukositis (M) } \\
\text { - Eine M-Behandlung reduziert das PI-Risiko }\end{array}$ \\
\hline & $\begin{array}{l}\text { Wennström \& } \\
\text { Derks } 2012\end{array}$ & $\begin{array}{l}\text { - Keratinisierte Mukosa bedeutet nicht zwingend } \\
\text { periimplantäre Gesundheit } \\
\text { - Sie steigert den Putzkomfort für den Patienten }\end{array}$ \\
\hline \multirow[t]{6}{*}{ Befundaufnahme } & $\begin{array}{l}\text { Schwarz \& } \\
\text { Becker } 2015\end{array}$ & $\begin{array}{l}\text { - Keine Symptome lassen nicht zwingend auf die } \\
\text { Gesundheit des Implantats schliessen } \\
\text { - Knochenabbau unterscheidet Mukositis von } \\
\text { Periimplantitis (cave: Remodeling) }\end{array}$ \\
\hline & $\begin{array}{l}\text { Serino et al. } \\
2013\end{array}$ & $\begin{array}{l}\text { - Suprakonstruktion bei Sondierung, wenn } \\
\text { möglich abnehmen }\end{array}$ \\
\hline & $\begin{array}{l}\text { Lang et al. } \\
2011 a\end{array}$ & $\begin{array}{l}\text { - Sondierungswerte in Relation zu Ausgangs- } \\
\text { messungen setzen (Kalibrierung) }\end{array}$ \\
\hline & $\begin{array}{l}\text { Schwarz et al. } \\
2007\end{array}$ & $\begin{array}{l}\text { - Mehr als die Hälfte der Knochendefekte sind } \\
\text { zirkumferentielle Klassse-I-Knochendefekte }\end{array}$ \\
\hline & $\begin{array}{l}\text { Casado et al. } \\
2011\end{array}$ & $\begin{array}{l}\text { - Mikrobiologische Diagnostik gegenwärtig nicht } \\
\text { indiziert }\end{array}$ \\
\hline & $\begin{array}{lll}\text { Faot et al. } \\
2015\end{array}$ & $\begin{array}{l}\text { - Bestimmung von Entzündungsmarkern noch } \\
\text { nicht indiziert }\end{array}$ \\
\hline \multirow[t]{2}{*}{ Therapie } & $\begin{array}{l}\text { Jepsen et al. } \\
2015\end{array}$ & $\begin{array}{l}\text { - M-Therapie basiert v.a. auf regelmässiger } \\
\text { mechanischer Reinigung } \\
\text { - Nicht alle Mukositiden heilen vorhersagbar ab } \\
\text { - Regelmässige Nachkontrollen }\end{array}$ \\
\hline & $\begin{array}{l}\text { Schwarz et al. } \\
2015 \text { a }\end{array}$ & - PI-Therapie: Frühe chirurgische Intervention \\
\hline
\end{tabular}




\begin{tabular}{|l|l|l|l|}
\hline & $\begin{array}{l}\text { Voraussagbare Dekontamination der } \\
\text { Implantat-Oberfläche unter Sicht }\end{array}$ \\
\hline
\end{tabular}

Tabelle 1: Zusammenfassung gesicherter evidenz-basierter Aussagen zur Mukositis und Periimplantitis.

\author{
Dr. Alex Solderer, Prof. Dr. Patrick R. Schmidlin
}

Klinik für Zahnerhaltung und Präventivzahnmedizihn

Bereich für Parodontologie und Periimplantäre Erkrankungen

Zentrum für Zahnmedizin der Universität Zürich

Plattenstrasse 11, CH-8032 Zürich

1. Schmidlin, P. R. 2018. The Strange Case of Peri-Implantology. Dent J (Basel) 6:

2. J., S. F. B. 2015. Periimplantäre Infektionen. Ein Update zur Epidemiologie, Ätiologie, Diagnostik, Prävention und Therapie. Quintessenz Implantologie. Quintessenz Implantologie 23: 1-13.

3. Lang, N. P., and J. Lindhe. 2015. Clinical Periodontology and Implant Dentistry, 2 Volume Set.

4. Lang, N. P., D. D. Bosshardt, and M. Lulic. 2011. Do mucositis lesions around implants differ from gingivitis lesions around teeth. J Clin Periodontol 38 Suppl 11: 182-187.

5. Salvi, G. E., M. Aglietta, S. Eick, A. Sculean, N. P. Lang, and C. A. Ramseier. 2012. Reversibility of experimental peri-implant mucositis compared with experimental gingivitis in humans. Clin Oral Implants Res 23: 182-190.

6. Heitz-Mayfield, L. J. 2008. Peri-implant diseases: diagnosis and risk indicators. J Clin Periodontol 35: 292-304.

7. Renvert, S., and I. Polyzois. 2015. Risk indicators for peri-implant mucositis: a systematic literature review. J Clin Periodontol 42 Suppl 16: S172-86.

8. Lang, N. P., T. Berglundh, and G. O. S. E. W. O. P. Working. 2011. Periimplant diseases: where are we now?--Consensus of the Seventh European Workshop on Periodontology. J Clin Periodontol 38 Suppl 11: 178-181.

9. Koldsland, O. C., A. A. Scheie, and A. M. Aass. 2011. The association between selected risk indicators and severity of peri-implantitis using mixed model analyses. J Clin Periodontol 38: 285-292.

10. Lin, G. H., H. L. Chan, and H. L. Wang. 2013. The significance of keratinized mucosa on implant health: A systematic review. Journal of Periodontology 84: 1755-1767.

11. Wennström, J. L., and J. Derks. 2012. Is there a need for keratinized mucosa around implants to maintain health and tissue stability. Clin Oral Implants Res 23 Suppl 6: 136146. 
12. Tabanella, G., H. Nowzari, and J. Slots. 2009. Clinical and microbiological determinants of ailing dental implants. Clin Implant Dent Relat Res 11: 24-36.

13. Serino, G., A. Turri, and N. P. Lang. 2013. Probing at implants with peri-implantitis and its relation to clinical peri-implant bone loss. Clin Oral Implants Res 24: 91-95.

14. Schwarz, F., M. Herten, M. Sager, K. Bieling, A. Sculean, and J. Becker. 2007. Comparison of naturally occurring and ligature-induced peri-implantitis bone defects in humans and dogs. Clinical Oral Implants Research 18: 161-170.

15. Golubovic, V., I. Mihatovic, J. Becker, and F. Schwarz. 2012. Accuracy of cone-beam computed tomography to assess the configuration and extent of ligature-induced periimplantitis defects. A pilot study. Oral \& Maxillofacial Surgery 16: 349-354.

16. Casado, P. L., I. B. Otazu, A. Balduino, W. de Mello, E. P. Barboza, and M. E. Duarte. 2011. Identification of periodontal pathogens in healthy periimplant sites. Implant Dent 20: 226-235.

17. Votta, I., S. Gartenmann, and P. R. Schmidlin. 2018. Das «Zero-Pocket-Konzept» im Rahmen der UPT. Swiss Dent J 128: 131-134.

18. Faot, F., G. G. Nascimento, A. M. Bielemann, T. D. Campão, F. R. Leite, and M. Quirynen. 2015. Can peri-implant crevicular fluid assist in the diagnosis of periimplantitis? A systematic review and meta-analysis. J Periodontol 86: 631-645.

19. Jepsen, S., T. Berglundh, R. Genco, A. M. Aass, K. Demirel, J. Derks, E. Figuero, J. L. Giovannoli, M. Goldstein, F. Lambert, A. Ortiz-Vigon, I. Polyzois, G. E. Salvi, F. Schwarz, G. Serino, C. Tomasi, and N. U. Zitzmann. 2015. Primary prevention of periimplantitis: managing peri-implant mucositis. J Clin Periodontol 42 Suppl 16: S152-7.

20. Salvi, G. E., and C. A. Ramseier. 2015. Efficacy of patient-administered mechanical and/or chemical plaque control protocols in the management of peri-implant mucositis. A systematic review. J Clin Periodontol 42 Suppl 16: S187-201.

21. Schwarz, F., A. Schmucker, and J. Becker. 2015. Efficacy of alternative or adjunctive measures to conventional treatment of peri-implant mucositis and peri-implantitis: a systematic review and meta-analysis. Int J Implant Dent 1: 22.

22. Solderer, A., A. Al-Jazrawi, P. Sahrmann, R. Jung, T. Attin, and P. R. Schmidlin. 2019. Removal of failed dental implants revisited: Questions and answers. Clin Exp Dent Res. 1-13. 